\title{
El métOdo comparativo en eCOLOGÍA VEGETAL
}

\author{
EduARDO MORALES \\ Instituto de Ecología, UNAM, Departamento de Ecología Evolutiva. \\ Apartado Postal 70-275. México, D.F. 04510, México. Correo electrónico: emorales@miranda.ecología.unam.mx
}

\begin{abstract}
Resúmen. En este trabajo se presenta una revisión sobre el empleo del método comparativo en ecología vegetal. En particular se hace referencia a la metodología estadística utilizada actualmente para analizar la variación en caracteres fenotípicos de distribución continua. El método realiza comparaciones entre especies para el análisis de la adaptación utilizando de manera explícita las filogenias y en su defecto, las taxonomías. De esta forma se reconoce que las especies no representan puntos independientes para realizar análisis estadísticos debido principalmente a que comparten caracteres por descender de un ancestro común. Este trabajo se divide en tres secciones, en la primera se presentan los diferentes enfoques de análisis del método comparativo contemporáneo, entre los principales se encuentran: i] el análisis de correlaciones evolutivas, ii] el análisis de la inercia filogenética, iii] el análisis de la estimación de los estados ancestrales de un carácter. Posteriormente se presentan evidencias del empleo de este método en ecología vegetal, tanto para análisis de investigaciones originales; como para re-análisis de información ya publicada. En la última sección se presenta el debate en torno al potencial del método comparativo en el estudio de la adaptación, y la discusión sobre el empleo de análisis comparativos versus análisis estadísticos convencionales.
\end{abstract}

Palabras clave: contrastes filogenéticamente independientes, estimación de estados ancestrales, método comparativo, modelo de autocorrelación.

Abstract. In this paper a review of the uses of the comparative method in plant ecology is presented. Particular attention is devoted to statistical methods that analyze variation in continuos phenotypic traits. The comparative method incorporates the phylogenetic relationships of the species in recognition that species usually do not provide independent points in statistical analysis because they share characteristics through descent from common ancestors. This review is divided in three sections. In the first one, the different statistical analysis that comprises the comparative method are presented, particular attention is devoted to: $i]$ Evolutionary correlations, ii] phylogenetic inertia, and iii] ancestral character estimation. The second section presents the different papers that had applied these different methodologies, in both, original or reanalyzed data. Finally, in the third section the use of comparative methods to study adaptation and the debate between the use of phylogenetically based statistical methods and conventional statistical analyses are discussed.

Key words: phylogenetic independent contrasts, ancestral states estimation, comparative method, autocorrelation model.

E

1 interés por hacer comparaciones y entender el proceso de adaptación en biología evolutiva no ha cesado. En los últimos 15 años se ha experimentado un cambio importante en el análisis de la información obtenida y en el re-análisis de la generada en el pasado con la introducción de los análisis estadísticos que incorporan la información filogenética de las especies a comparar (Brooks y Mc Lennan 1991;
Harvey y Pagel 1991). Este tipo de análisis se conoce genericamente como el Método Comparativo.

El método comparativo puede definirse como el uso de comparaciones entre especies para proponer o probar hipótesis adaptativas y otros fenómenos evolutivos (Futuyma 1998). El mismo autor considera que el método comparativo es un medio poderoso para inferir la significancia adaptativa de un carácter de- 
terminado, el cual toma ventaja de "experimentos naturales" determinados por procesos de evolución convergente. Sin embargo, aunque el término adaptación ha estado en debate por largo tiempo (ver Rose y Lauder 1996) la mayoría de los estudiosos del tema consideran que una adaptación representa un fenotipo que muestra mayor adecuación que otro fenotipo en el mismo ambiente. Adicionalmente, los estudiosos de la sistemática filogenética argumentan que el fenotipo debió haber sido moldeado evolutivamente por la selección natural para cumplir el papel que actualmente deempeña; éste ha sido definido como el enfoque histórico.

Uno de los aspectos más importantes en el desarrollo del método comparativo es el reconocimiento de que las especies a comparar no son muestras estadísticamente independientes entre sí, debido a que comparten caracteres por descender de ancestros comunes (Felsenstein 1985; Pagel y Harvey 1988). Con base en lo anterior, resulta importante incluir la información filogenética de las especies en los análisis estadísticos comparativos.

Wanntorp (1983), fue seguramente quien realizó el primer estudio en ecología vegetal que incorporó información de las relaciones filogenéticas al intentar explicar el valor adaptativo de presentar o no hojas caducifolias en diferentes especies de encinos europeos. Sobreponiendo a la topología de los encinos el carácter de ser deciduo o no, y los diferentes ambientes en que se distribuyen las especies, en un ejercicio similar al mapeo de caracteres en sistemática filogenética, mostró que la retención de hojas es el estado ancestral del carácter y está asociado a ambientes tropicales y mediterráneos. Por lo tanto, el ser deciduo es el estado derivado y se considera que es una adaptación a ambientes fríos. (ver figural)

Givnish (1987) compara la morfología foliar, para ilustrar el papel relevante de las comparaciones ecológicas, biogeográficasy filogenéticas para generar y comparar hipótesis respecto al valor adaptativo de la variación morfológica.

Las preguntas más comunes que se intentan responder con la aplicación de la metodología comparativa están relacionadas con: i] la evolución correlacionada de caracteres; ii] la estimación del efecto de las relaciones filogenéticas sobre el carácter evaluado (i.e. inercia filogenética), y iii] la estimación de los estados ancestrales de caracteres fenotípicos.

En el presente trabajo, presentaré una revisión sobre los aspectos más importantes de este tipo de análisis, y discutiré los diferentes usos del método comparativo en ecología y evolución. La presentación de la información la dividiré en tres secciones. En la primera presentaré los diferentes modelos empleados en los estudios comparativos, en particular me enfocaré en los métodos desarrollados para analizar caracteres continuos. No incluiré análisis para caracteres discretos porque considero que éstos han sido trata-

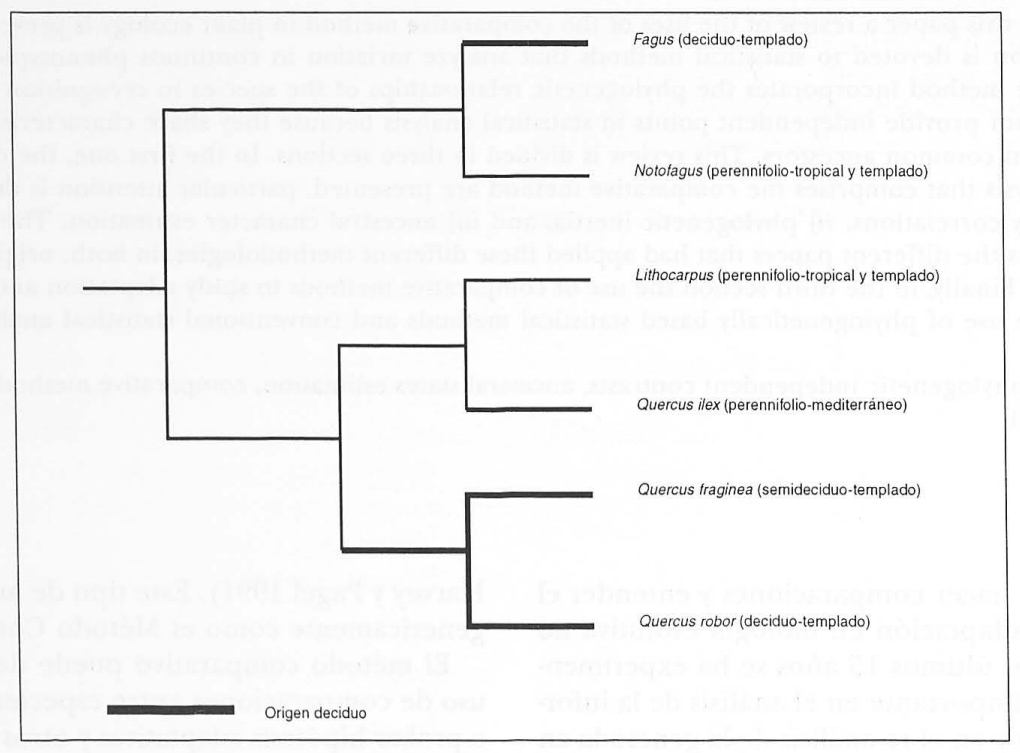

Figura 1. Cladograma simplificado de la familia Fagaceae que sugiere que que el carácter deciduo ha evolucionado dos veces en el grupo de los encinos de zonas templadas (modificado de Wanntorp 1983). 
dos con amplitud en otros trabajos (p.ej. Ridley y Grafen 1996). En la segunda, incluiré los trabajos que en biología vegetal han empleado alguno de los diferentes modelos, ya sea para analizar información original o re-analizar información existente en la literatura, incluida mi propia experiencia. Finalmente discutiré una serie de reflexiones sobre los avances que pueden presentar estas metodologías al realizar estudios en ecología y evolución, así como una serie de consideraciones sobre la aplicación de esta herramienta estadística.

\section{Los Métodos}

Contrastes filogenéticamente independientes. Este modelo fue derivado por Felsenstein (1985) y principalmente permite analizar correlación evolutiva entre pares de caracteres. Se denomina el análisis de contrastes filogenéticamente independientes (PIC por sus siglas en inglés) y fue derivado inicialmente para evitar el problema de la no independencia estadística cuando se realizan comparaciones entre especies hermanas. La idea central en este método es que el cambio que ocurre posterior a un evento de especiación es independiente entre especies hermanas. Supongamos una hipótesis filogenética con ocho especies (A,B,C,D,E,F,G,H) de la cual conocemos datos sobre el tamaño y la longevidad (ver figura 2). Las especies A y B, C y D, E y F, $\mathrm{G}$ y $\mathrm{H}$; forman cuatro pares de especies hermanas.
El modelo propone que los cambios que ocurren entre A y B después del evento de especiación son independientes de los cambios ocurridos en C y D después de la especiación aunque los cuatro taxa compartan un ancestro común. Así, si medimos dos caracteres $\mathrm{X}$ y $\mathrm{Y}$ (tamaño y longevidad) para cada especie, entonces la diferencia entre $\mathrm{A}$ y $\mathrm{B}$ para el carácter $\mathrm{X}$ es independiente de la diferencia entre C y D para el mismo carácter X. Las diferencias así calculadas se denominan contrastes independientes. El método puede ser aplicado para contrastes obtenidos sobre toda la topología y el valor para un carácter en nodos "inferiores" como el promedio de los valores de los descendientes.

El método asume que la evolución de un carácter fenotípico puede describirse empleando un modelo de movimiento Browniano, el cual es un proceso aleatorio en el que el cambio evolutivo que ocurre en los atributos fenotípicos durante cualquier intervalo de tiempo, se encuentra normalmente distribuido y es independiente del estado del fenotipo en el inicio del intervalo de tiempo (p.ej. cualquier bifurcación sobre la topología). Asimismo, bajo este supuesto la longitud de las ramificaciones en cualquier evento de especiación sobre la topología será la misma. Los contrastes son las variables que son sujeto del análisis, de correlación en este caso, y aunque bajo estas premisa los datos de las especies no son independientes, las diferencias o contrastes entre pares de espe-

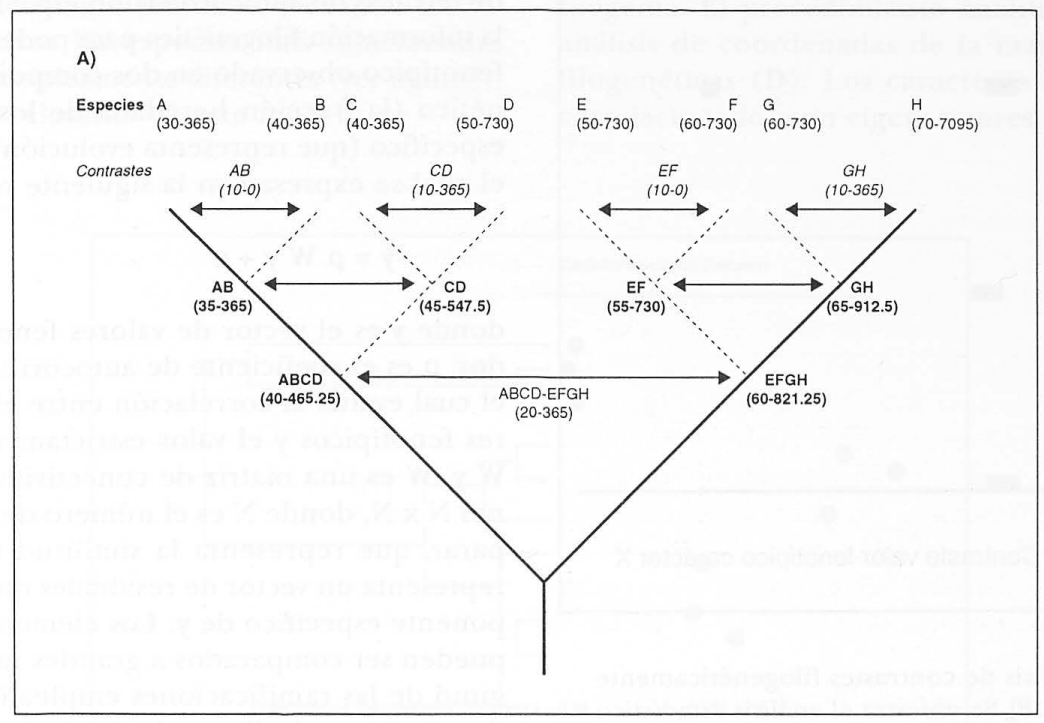

Figura 2. Análisis de contrastes filogenéticamente independientes. a] Se muestran los valores observados y las relaciones filogenéticas para las especies analizadas. En negritas se muestran los valores estimados para los diferentes nodos y en itálicas se muestran los valores para los contrastes. 
cies de la correspondiente filogenia sí lo son. El número de contrastes posibles para una filogenia dada es $\mathrm{N}-1$, siendo $\mathrm{N}$ el número de especies en dicha filogenia (ver figura 2).

El modelo de "contrastes" es el método más utilizado en ecología vegetal para intentar responder a dos preguntas básicas: $i]$ ¿qué caracteres se encuentran correlacionados entre sí y con el ambiente? y ii] ¿son estas correlaciones el resultado de descendencia común o son convergencias evolutivas?

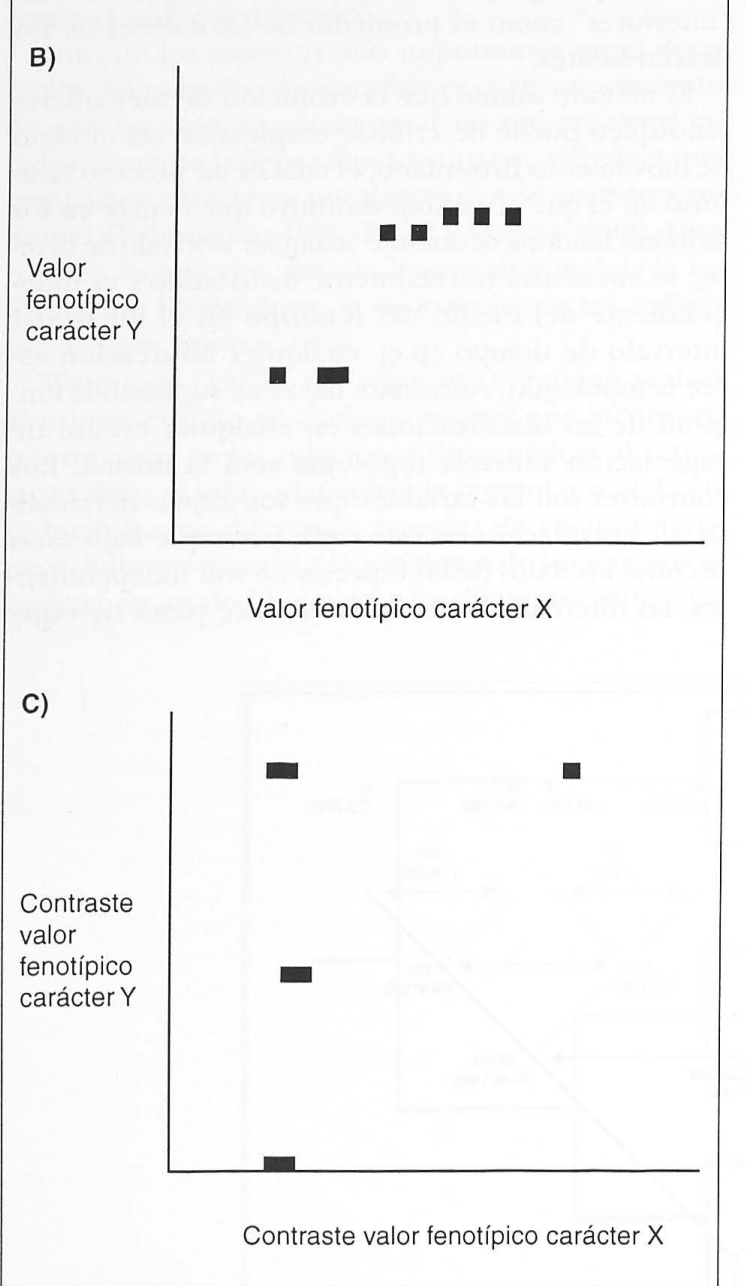

Figura 2 b. Análisis de contrastes filogenéticamente independientes. $B$ ] Se muestra el análisis estadístico convencional que señala una fuerte correlación positiva y $C$ ] La correlación empleando la técnica de contrastes muestra que la correlación puede resultar espuria.
La aceptación y la aplicación de este método ha aumentado considerablemente, por lo que es necesario e importante comparar los resultados obtenidos en los estudios empleando el método comparativo, con los análisis estadísticos convencionales de correlación considerando los caracteres fenotípicos de las especies sin ninguna transformación. Esta comparación nos permitiría: i] validar o reinterpretar conclusiones de estudios previos en los que se hicieron comparaciones sin considerar la información filogenética de las especies a comparar y ii] también nos permitiría justificar estudios comparativos en los que no se incluyeran las relaciones filogenéticas, cuando dicha información no se encuentra disponible aún. Los trabajos de Kelly y Purvis (1993) y Franco y Silvertown (1996) son un excelente ejemplo respecto a los dos apartados mencionados (ver los detalles en evidencias de estudios comparativos).

Análisis de autocorrelación filogenética. Estos modelos están diseñados particularmente para el análisis cuantitativo de los efectos filogenéticos y específicos en los atributos fenotípicos de diferentes especies. El valor de un carácter fenotípico dado puede dividirse en su valor filogenético, heredado de sus ancestros a través de su filogenia y su valor específico, resultado de evolución independiente. Lo anterior permite obtener una evaluación cuantitativa de la inercia filogenética para un atributo en un linaje determinado. Modelo de autoregresión. Este modelo fue desarrollado por Cheverud et al. (1985), quienes sugirieron el uso de técnicas de autocorrelación espacial, que emplean la información filogenética para poder dividir un valor fenotípico observado en dos componentes: el filogenético (la fracción heredada de los ancestros) y el específico (que representa evolución independiente) el cual se expresa con la siguiente relación:

$$
\mathbf{y}=\rho \mathbf{W} \mathbf{y}+\varepsilon
$$

donde y es el vector de valores fenotípicos observados, $\rho$ es el coeficiente de autocorrelación a estimar el cual evalúa la correlación entre el vector de valores fenotípicos y el valor estrictamente filogenético W y. W es una matriz de conectividad de dimensiones $\mathrm{N}$ x N , donde $\mathrm{N}$ es el número de especies a comparar, que representa la similitud filogenética y $\varepsilon$ representa un vector de residuales que indica el componente específico de y. Los elementos de la matriz pueden ser comparados a grandes rasgos con la longitud de las ramificaciones empleadas en el análisis de contrastes y la diagonal principal se representa con valores igual a cero. Sin embargo, los elementos fuera de la diagonal pueden ser calculados en más de una forma y esto depende del tipo de enfoque, ya sea 
empírico o de simulación. En general existen dos opciones para determinar los elementos de W: el inverso de las distancias pareadas entre especies (Westnet 1995) o el número de nodos que separan pares de especies (Miles y Dunham 1992). Para estimar el modelo de regresión se emplea un modelo de máxima verosimilitud, puesto que la variable independiente y aparece en ambos lados de la ecuación. Gittleman y Kott (1990) hicieron una extensión del modelo, que permite estimar un parámetro $(\alpha)$ el cual transforma las distancias pareadas de $\mathbf{W}$, el cual es similar a la transformación de longitud de las ramas que puede ser empleada en el análisis de contrastes.

El método de autocorrelación filogenética presenta dos aspectos relevantes. Primero, el valor estimado de los residuales $\varepsilon$ debe de estar libre de los efectos filogenéticos y por lo tanto ser independiente estadísticamente. Si esto se cumple puede ser utilizado en análisis estadísticos convencionales. En este sentido los valores del vector $\varepsilon$ serían análogos a los contrastes propuestos por Felsenstein (1985). La diferencia radica en que mientras los contrastes retienen el 100\% de la varianza original de los datos, los residuales del modelo de autocorrelación corresponden sólo a una fracción de la varianza total.

Segundo, el coeficiente de autocorrelación ( $\rho$ ), el cual puede obtener valores entre -1 y +1 , refleja el grado de similitud entre especies relacionadas y puede ser probada su significancia estadística. Valores positivos de $\rho$ indican que especies cercanamente relacionadas son fenotípicamente similares, y cuando los valores son negativos las especies más relacionadas tienden a ser fenotípicamente diferentes (ver figura3). Otro estadístico relevante de este modelo es:

$$
\mathbf{R}^{2}=1-(\text { varianza de } \varepsilon / \text { varianza de } \mathbf{y})
$$

el cual representa la proporción de la varianza que está determinada por la filogenia.

La relevancia de este método radica en que además de ser el primer modelo para estimar los efectos filogenéticos sobre los atributos fenotípicos, es el más comúnmente utilizado para responder este tipo de preguntas en ecología y evolución. Además, su empleo se facilita puesto que se ha implementado el programa MRHO3, el cual permite el análisis comparativo de hasta 150 especies. Aunque no han sido formalizados, Martins y Hansen (1996) consideran que algunos de los supuestos importantes del modelo de autoregresión son los siguientes: $i]$ la variación intraespecífica es ilegible o incorporada dentro del análisis, ii] se conocen las relaciones filogenéticas de las especies a comparar, iii] cada fenotipo puede ser descrito como una función lineal de los otros fenotipos de la filogenia, $i v]$ cada fenotipo puede ser descrito como la suma de sus componentes filogenético y específico y $v$ ] sólo el componente específico e puede ser empleado para posteriores análisis.

Modelo de "eigenvectores" para estimar la inercia filogenética (PVR). Este método fue propuesto por Diniz-Filho et al. (1998) y ha sido desarrollado también para evaluar efectos filogéneticos y específicos. El modelo requiere una hipótesis filogenética y un atributo fenotípico evaluado sobre todas las especies de dicha filogenia. El procedimiento analítico inicia con un análisis de coordenadas de la matriz de distancias filogenéticas (D). Los caracteres bajo análisis son correlacionados con eigenvectores resultantes de un

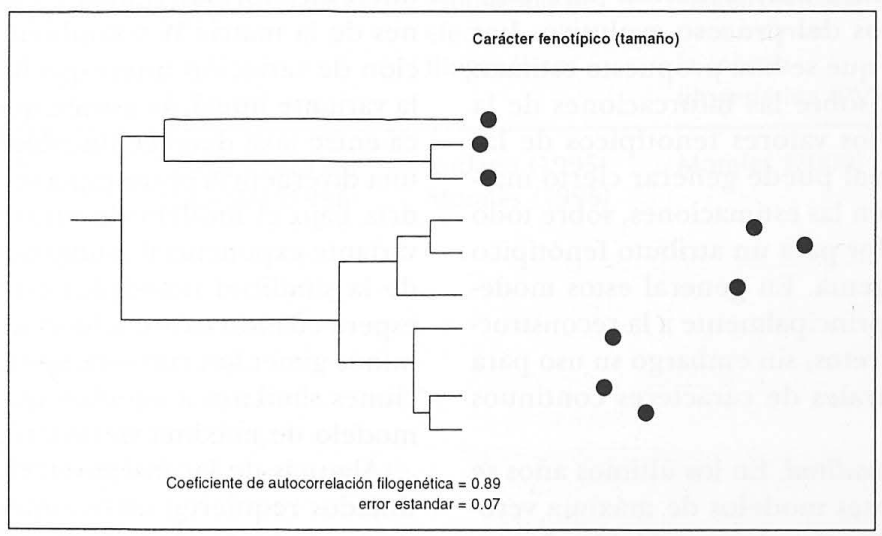

Figura 3. Análisis de autocorrelación filogenética que muestra que las especies similares tienden a tener una respuesta similar $\rho=0.89$. 
modelo de "vara-rota" de tal forma que los valores estimados representan las tendencias filogenéticas de los caracteres y los residuales expresan el componente de evolución independiente para cada especie. La diferencia de este modelo respecto al propuesto por Cheverud et al. (1985), radica en el mejor desempeno estadístico cuando la correlación filogenética es baja o el tamaño de muestra es muy pequeño para detectarlo. Formalmente el modelo se expresa así: $\mathbf{Y}=$ $\mathbf{X} \beta+\varepsilon$. Donde $\mathbf{Y}$ es la variable de respuesta, el valor del caracter fenotípico observado, $\mathbf{X}$ son los eigenvectores de la matriz de distancias transformada y $\beta$ es el vector con coeficientes de regresión estimados con la técnica de mínimos cuadrados. En este modelo, el componente filogenético está dado por los valores estimados de Y (Y') y el componente específico está dado por los residuales de la regresión $\left(\varepsilon=\mathbf{Y}-\mathbf{Y}^{\prime}\right)$, los cuales contienen la fracción de la varianza que no se encuentra determinada por la estructura filogenética. El modelo emplea una regresión múltiple que permite la obtención de un valor de $\mathbf{R}^{2}$ (coeficiente de determinación) que puede ser utilizado como un estimador de la magnitud de la inercia filogenética en los atributos fenotípicos evaluados.

Modelos para la estimación de estados ancestrales. Este procedimiento es muy utilizado en sistemática filogenética y se emplea para determinar cambios en los estados de caracteres, así como para conocer el número de veces que a lo largo de una filogenia un carácter ha surgido de manera independiente. En ausencia de cualquier evidencia paleontológica, estimar el estado ancestral de un atributo fenotípico determinado, es de gran relevancia para entender patrones de diversificación ecológica, entre otros atributos. La reconstrucción de los estados ancestrales es una herramienta analítica muy poderosa para estudiar diferentes aspectos del proceso evolutivo. Los modelos comparativos que se han propuesto estiman los valores ancestrales sobre las bifurcaciones de la filogenia, a partir de los valores fenotípicos de las especies actuales, lo cual puede generar cierto margen de incertidumbre en las estimaciones, sobre todo cuando se estima el valor para un atributo fenotípico en la raíz de una filogenia. En general estos modelos han sido aplicados principalmente a la reconstrucción de caracteres discretos, sin embargo su uso para estimar estados ancestrales de caracteres continuos es ahora más común.

Modelo de máxima verosimilitud. En los últimos años se han propuesto diferentes modelos de máxima verosimiltud (traducción libre de maximum-likelihood) para reconstruir estados ancestrales de carácter en atributos fenotípicos discretos (Schluter 1995, Yang et al. 1995,
Koshi y Goldstein 1996 y Zhang y Nei 1997). Estos análisis emplean un modelo explícito de evolución fenotípica para evaluar las probabilidades de todos los posibles cambios en los estados de los caracteres, en todos los nodos del árbol filogenético. Además de emplear un modelo de evolución particular, para el cálculo de dichas probabilidades se incluye la distribución de los estados de carácter en los taxa terminales, la tasa de evolución de los mismos y la longitud de las ramas del árbol.

Recientemente Schluter et al. (1997) propusieron una extensión al modelo de máxima verosimilitud para poder ser empleado en el análisis de caracteres continuos. El modelo supone que los caracteres evolucionan conforme al proceso de movimiento Browniano. Los supuestos del modelo son los siguientes: i] la probabilidad de cambio en cualquier punto de la filogenia depende únicamente del estado del carácter en ese punto y no en los valores previos del mismo, ii] las transiciones en cualquier rama del árbol son independientes de cualquier otro cambio en el árbol lo que puede permitir una estimación no parsimoniosa y iii] las tasas de cambio son constantes en el tiempo.

Modelo lineal generalizado. Martins y Hansen (1997) desarrollaron un modelo para estimar estados ancestrales y errores estandar en caracteres continuos. Este método emplea el modelo $\mathbf{A}=\mathbf{W} \mathbf{Y}$ para estimar los estados ancestrales, donde $\mathbf{Y}$ es el vector de los atributos fenotípicos de las especies, $W$ es la matriz que describe las relaciones filogenéticas y el modelo de evolución fenotípica y $\mathbf{A}$ es un vector de estimados de los estados ancestrales.

El modelo permite incorporar diferentes supuestos evolutivos como selección estabilizadora y evolución en ambientes fluctuantes, al ponderar de dos diferentes formas (lineal y exponencial) las relaciones de la matriz $\mathbf{W}$ y también permite la incorporación de variación interespecífica. Cuando se emplea la variante lineal, se asume que la similitud fenotípica entre taxa decrece linealmente, lo que determina una divergencia entre especies como la que se obtendría bajo el modelo de movimiento Browniano. La variante exponencial asume decaimiento exponencial de la similitud fenotípica entre especies, lo que se espera cuando existe selección estabilizadora. En términos generales con este modelo se obtienen estimaciones similares a aquellas que se obtendrían con el modelo de máxima verosimilitud.

Algunas de las interpretaciones de los valores estimados requieren otros supuestos, por ejemplo, algunas de las tendencias de la dirección del cambio pueden violar el supuesto de movimiento browniano y podrían alterar la utilidad de los estimados para 
establecer patrones de cambio y estos estar sesgados a valores muy altos o bajos, según sea el caso.

\section{Evidencias de estudios comparativos}

En esta sección incluiré los estudios que han empleado metodologías estadísticas comparativas tanto para investigaciones originales como para aquellos trabajos en que se analiza nuevamente información ya publicada.

En general el uso del método comparativo, con las características que he señalado en los párrafos anteriores, no se encuentra muy difundido en la investigación botánica. Los estudios de Wanntorp (1983) y Givnish (1987) son los que podríamos considerar pioneros en incorporar las relaciones filogenéticas para realizar comparaciones en ecología vegetal. Sin embargo, dichos estudios no incorporaban análisis comparables a los mostrados en la sección anterior, eran más bien descriptivos con algunas hipótesis sobre la posibilidad de determinar el valor adaptativo de los atributos fenotípicos evaluados. Lo anterior, no significa o intenta demeritar la gran contribución de ambos estudios a la ecología vegetal, es más bien un reconocimiento.

Formalmente con todos los riesgos que esto implica considero que este tipo de análisis se han vuelto "más difundidos y empleados" a partir de la discusión organizada en 1996 por J. Silvertown, M. Francoy J. L. Harper titulada: Plant life histories: ecological correlates and phylogenetic constraints la cual ha sido revisada y criticada por Mazer (1998). Previo a esta discusión, los estudios que incorporaban información taxonómica de las especies a comparar, básicamente evaluaban, vía análisis de varianza anidados los porcentajes de varianza explicados a diferentes niveles taxonómicos para el carácter estudiado.

\section{Contrastes filogenéticamente independientes}

Este tipo de análisis comparativos es el que se encuentrá más difundido en ecología vegetal (ver tabla 1). Considero que sucede así porque históricamente se han buscado correlaciones entre caracteres, y entre éstos y el ambiente para interpretarlas ecológica o evolutivamente. Sin embargo, ahora al emplear el método comparativo la pregunta es explícita: ¿las correlaciones entre atributos fenotípicos reflejan patrones históricos de cambios evolutivos correlacionados? Más aún, en algunos estudios se intenta explicar en términos adaptativos la presencia de correlaciones.

Existe un conjunto de trabajos interesantes realizados por Kelly y colaboradores (Kelly y Purvis 1993, Kelly y Beerling 1995, Kelly y Woodward 1995), en los que se reanalizan patrones ecológicos, y principalmente se desea constatar si las conclusiones originales pueden mantenerse cuando es considerada la similitud taxonómica. Formalmente, la metodología empleada no es idéntica a la propuesta en el modelo de Felsenstein (1985), más bien es una extensión de éste, debido a que los contrastes que se obtienen no son analizados con un modelo de correlación, sino con

Tabla 1. Estudios en ecología vegetal que han empleado análisis comparativos.

\begin{tabular}{|c|c|c|c|c|}
\hline $\begin{array}{l}\text { Análisis de contrastes } \\
\text { filogenéticamente } \\
\text { independientes }\end{array}$ & $\begin{array}{l}\text { Modelo de } \\
\text { autocorrelación }\end{array}$ & $\begin{array}{l}\text { Modelo para la } \\
\text { estimación de } \\
\text { efectos } \\
\text { filogenéticos }\end{array}$ & $\begin{array}{l}\text { Modelo de } \\
\text { "eigenvectores" } \\
\text { para estimar } \\
\text { la inercia } \\
\text { filogenética (PVR) }\end{array}$ & $\begin{array}{l}\text { Modelos para } \\
\text { la estimación de } \\
\text { estados } \\
\text { ancestrales }\end{array}$ \\
\hline $\begin{array}{l}\text { Armstrong y Westoby (1993) } \\
\text { Kelly y Purvis (1993) } \\
\text { Kelly (1995) } \\
\text { Kelly y Beerling (1995) } \\
\text { Kelly y Woodward (1995) } \\
\text { Beerling y Kelly (1996) } \\
\text { Crawley et al. (1996) } \\
\text { Franco y } \\
\text { Silvertown (1996) } \\
\text { Kelly y Woodward (1996) } \\
\text { Rees (1996) } \\
\text { Silvertown y Dodd (1996) } \\
\text { Ezcurra et al (1997) } \\
\text { Ackerly y Donoghue (1998) }\end{array}$ & $\begin{array}{l}\text { Jordano (1995) } \\
\text { Morales (1996) }\end{array}$ & $\begin{array}{l}\text { Jordano (1995) } \\
\text { Morales (1996) }\end{array}$ & Morales (2000) & $\begin{array}{l}\text { Ackerly y } \\
\text { Donoghue (1998) } \\
\text { Morales (2000) }\end{array}$ \\
\hline
\end{tabular}


una prueba de signos y las filogenias con las que se cuenta son obtenidas a partir de información taxonómica. En el primer trabajo en que emplean esta técnica, Kelly y Purvis (1993) reanalizan la hipótesis de Foster y Janson (1985), sobre la correlación existente entre el tamaño de la semilla de ciertas especies de árboles tropicales con el área de los claros que colonizaban. Los autores concluyeron que las especies de árboles con semillas de tamaño mayor tendían a establecerse en claros de tamaño pequeño o sombreados. Al ser incluída la información filogenética de las especies a comparar, dicha conclusión no podía ser sostenida y determinaron que no existía correlación entre el tamaño de la semilla y el del claro a colonizar. Con la misma metodología (Kelly y Beerling 1995), reanalizaron la propuesta de Salisbury (1927) sobre la variación en la densidad estomática de especies de diferentes formas de vida en comunidades boscosas. La propuesta original indicaba que la densidad estomática mayor era para especies árboreas, posteriormente para arbustos, en tercer lugar para especies leñosas, luego para especies de hierbas de los límites del bosque y finalmente para hierbas del sotobosque. Con el análisis de contrastes las conclusiones originales no se mantienen y la mayor densidad estomática corresponde a las especies de arbustos, posteriormente árboles y finalmente hierbas. Cuando se analizan los grupos de especies para un mismo hábitat, no se encontraron diferencias entre formas de vida. Finalmente Kelly y Woodward (1995), reanalizan las relaciones existentes entre la composición de isotopos de carbón $\left(\delta^{13} \mathrm{C}\right)$ y la distribución altitudinal y latitudinal de las especies de plantas. Los autores encontraron un efecto positivo de la altitud, pero no encontraron efecto alguno de la latitud como previamente se había reportado. Concluyen que las diferencias en la composición de la atmósfera (presiones parciales de $\mathrm{CO}_{2}$ y $\mathrm{O}_{2}$ ) representadas por la altitud, son suficientes para explicar las diferencias observadas en la composición de isotopos de carbón $\left(\delta^{13} \mathrm{C}\right)$.

Armstrong y Westoby (1993), compararon con un análisis de contrastes independientes, el efecto de eliminar $95 \%$ del tejido fotosintético en el crecimiento y supervivencia de 40 especies de plántulas de angiospermas australianas. Reportan que las especies con tamaño de semilla mayor presentan una supervivencia más alta en comparación a las especies con semilla pequeña. Concluyen que las semillas de tamaño mayor pueden verse favorecidas en situaciones en que las plántulas experimenten déficit de carbono en etapas tempranas de desarrollo.

Kelly (1995), analizó desde una perspectiva filogenética, las relaciones existentes entre el tamaño de la semilla y la altura de las plantas, respecto a su es- tadio sucesional y sus agentes dispersores en más de 200 especies de árboles tropicales en las selvas peruanas. Con el análisis de contrastes determinó que no existe correlación evolutiva entre el tamaño de la semilla y el estado sucesional y que existe parcialmente entre la altura de las plantas y el estado sucesional. Finalmente muestra que la masa de las semillas y la estaturas de las especies si está evolutivamente correlacionada. Concluye que la evidencia que presenta no coincide con la propuesta original (Foster y Janson 1985) de que la masa de las semillas está correlacionada con el estadio sucesional y el síndrome de dispersión.

Jordano (1995) evaluó la relación entre la variación fenotípica de frutos de angiospermas y sus dispersores. El análisis de contrastes sugiere dos patrones a este estudio. Primero, no se muestra evolución correlacionada entre el tipo de dispersión y los atributos fenotípicos de los frutos. Segundo, el diámetro de los frutos mostró una asociación con el tipo de dispersor, mostrando la tendencia de que los mamíferos funcionan como los principales agentes de dispersión. Concluye que la evidencia encontrada demuestra que el incremento (evolutivo) en el tamaño del fruto se encuentra asociado a síndromes de dispersión mixtos y de mamíferos.

Franco y Silvertown (1996), efectúan un análisis de contrastes para evaluar correlaciones entre ocho caracteres de historia de vida en ochenta y tres especies de plantas superiores. Entre los resultados más importantes destaca el que la mayoría de las correlaciones encontradas al analizar los datos fenotípicos, sin considerar la filogenia de las especies, se mantienen al incluir la información filogenética y taxonómica. La principal diferencia entre dichas correlaciones es la intensidad de la pendiente, las correlaciones fenotípicas sin considerar la filogenia presentan la mayor pendiente, seguidas de las que incoporan la filogenia y por último las que incluyen la taxonomía. También es relevante apuntar que el sentido de la correlación se mantiene independientemente del tipo de datos analizados (con y sin filogenia ó taxonomía). Los autores concluyen que: i] la existencia de una disyuntiva entre crecimiento y reproducción y ii] alcanzado el estado adulto la mortalidad es constante e independiente de la reproducción.

Silvertown y Dodd (1996) reanalizaron las hipótesis de asignación a la reproducción diferencial en función del estadio sucesional (Abrahamson 1979) y la apariencia de las especies vegetales ante sus herbívoros (Feeney 1976). El análisis de contrastes apoya la hipótesis de Abrahamson (1979) de que las especies anuales y de estadios de sucesión temprana presentan una mayor asignación a la reproducción 
respecto a las especies perennes y de estadios de sucesión tardíos. La hipótesis de apariencia (Feeney 1976) sugiere que las especies vegetales que son más aparentes a los herbívoros invierten más en taninos para su defensa, mientras que las menos aparentes asignan más a la producción de compuestos tóxicos cómo los alcaloides. La conclusión obtenida con el análisis de contrastes es la misma que la del trabajo original, aunque los autores afirman que los resultados no se contraponen con teorías que sugieren predicciones similares.

Ezcurra et al. (1997), evaluaron la evolución de la morfología foliar de Chuquiraga (Asteraceae) en relación con el clima. Con el análisis de contrastes evaluaron si la reducción del ancho de las hojas se presentaba en estas especies debido a una adaptación a climas desérticos. Los resultados muestran que la reducción en el tamaño de la hoja ha ocurrido independientemente en dos ocasiones durante la evolución del grupo y que la reducción en el tamaño de la hoja se correlacionaba con incrementos en la temperatura. Concluyen que la evolución en la morfología de la hoja es debida a efectos climáticos relativamente recientes.

Ackerly y Donoghue (1998), analizaron en 17 especies del género Acer la evolución de 32 caracteres fenotípicos con el objetivo de determinar si las, correlaciones fenotípicas observadas entre las especies reflejaban patrones históricos de cambios evolutivos correlacionados. Asimismo, evaluaron la regla de Corner (Hallé et al. 1978 en Ackerly y Donoghue 1998) la cual predice que los tamaños de las hojas y de las inflorecencias se encuentran positivamente correlacionados en la evolución de la morfología de especies de árboles e inversamente correlacionada con la densidad de ramas en la copa. El análisis de contrastes en relación a la regla de Corner es significativo y en el mismo sentido de la predicción. En contraste, no se obtuvieron correlaciones entre el tamaño de la hoja y la amplitud de la copa y entre ésta y el índice de área foliar, como anteriormente se había propuesto para estudios de alometría y de historia de vida en árboles.

\section{Análisis de autocorrelación filogenética}

Existen estudios en ecología vegetal que sugieren que las restricciones filogenéticas son importantes (ver Jordano 1995 para una revisión), sin embargo han existido pocos intentos en los que realmente se haya cuantificado esta inercia. Los métodos de autocorrelación al separar los componentes de varianza filogenética y específica de un atributo fenotípico, permiten estimar dicha similitud histórica.
Jordano (1995), evaluó con el método de Cheverud et al. (1985) explícitamente qué fracción de la varianza fenotípica total en frutos de angiospermas (910 especies) es explicada debido a compartir ancestros comúnes. Los caracteres evaluados los dividió en caracteres de diseño $\mathrm{N}=7$ (tamaño del fruto, masa del fruto, semillas por fruto, entre otros) y de contenido nutricional $\mathrm{N}=9$ (proporción de agua, de lípidos, por mencionar algunos). Obtuvo autocorrelaciones significativas para los 7 caracteres de diseño analizados y para cuatro caracteres de contenido nutricional, lo cual indica un efecto de la filogenia. La varianza explicada por la filogenia varía de $7.65 \%$ a $31.56 \%$ en caracteres de diseño y de $0.14 \%$ a $23.12 \%$ en caracteres de contenido nutricional. Se analiza también las trayectorias que presentan los correlogramas filogenéticos (Gittleman y Kot 1990) para los dos diferentes grupos de caracteres. Se observa en general que los atributos de diseño presentan una tendencia a decrecer conforme las especies se alejan en la jerarquía taxonómica, conforme lo predice el modelo de autocorrelación. Los caracteres de contenido nutricional no presentan esta tendencia de una manera tan contundente. Concluye que las diferencias entre los síndromes de dispersión se diluyen al incorporar los efectos filogenéticos, lo que revela que los síndromes de dispersión de semillas no pueden interpretarse totalmente como adaptaciones actuales a los dispersores de semillas.

El segundo trabajo que emplea el análisis de autocorrelación es el que realicé para evaluar la inercia filogenética y la fracción de la varianza asociada a las relaciones filogenéticas en caracteres demográficos y ecológicos en el género Tithonia (Asteraceae) Morales (1996 y 2000). El objetivo principal del estudio consistió en evaluar con diferentes métodos de autocorrelación (Cheverud et al. 1985, Gittleman y Kot 1990, Diniz-Filho et al. 1998), la fracción de la varianza asociada a las relaciones genealógicas para probar la hipótesis de que los atributos morfológicos presentan mayor inercia filogenética que los demográficos. Los resultados de los análisis de autocorrelación (Cheverud et al. 1985 y Diniz-Filho et al. 1998), apuntan en este sentido. Del total de caracteres evaluados (6 morfológicos o de diseño y 8 demográficos o de historia de vida), siete de ellos (cinco morfológicos y dos demográficos) presentan coeficientes de autocorrelación estadísticamente significativos. Las fracciones de la varianza explicadas por las relaciones filogenéticas oscilan entre el $30 \%$ y el $80 \%$ para los caracteres morfológicos y entre el $53 \%$ y $69 \%$ para los caracteres demográficos. Sin embargo, al hacer las proyecciones de los correlogramas filogenéticos, los resultados no son coincidentes con los anteriores 
análisis. De siete caracteres con una relación clara entre el índice I de Moran y la distancia filogenética, tres de ellos son morfológicos y cuatro son demográficos. La diferencia en los resultados no me permite aceptar o rechazar la hipótesis, sin embargo estas diferencias son útiles para discutir los alcances de los diferentes tipos de análisis en la biología comparada (ver sección de discusión).

\section{Modelos para la estimación de estados ancestrales}

Este tipo de análisis sólo ha sido aplicado un par de ocasiones en estudios que emplean el método comparativo (Ackerly y Donoghue 1998 y Morales 2000). En el primer trabajo la reconstrucción de los estados ancestrales se realiza con una metodología similar a la técnica de parsimonia, mientras que en mi trabajo empleé la rutina de máxima verosimilitud de Schluter et al. (1997).

Ackerly y Donoghue (1998), definieron el índice de convergencia cuantitativa para, precisamente evaluar la convergencia evolutiva en 32 caracteres fenotípicos para 17 especies del género Acer. Este procedimiento permite estimar los estados de los caracteres en diferentes bifurcaciones del árbol filogenético incluida la raíz y estos valores pueden representar estimaciones de los estados ancestrales de los caracteres. Los autores ejemplifican este análisis con los cambios evolutivos en el tamaño de la hoja y resulta interesante observar la diferenciación del carácter sugerida por este método. Se observa que el tamaño de la hoja varía en doce taxa, la tendencia al incremento del atributo se observa en siete taxa y la disminución en cinco. Al calcularse el índice de convergencia, los autores sugieren un nivel muy alto de evolución convergente en el tamaño de la hoja.

En los estudios que he realizado con el género Tithonia, también he obtenido estimaciones de los estados ancestrales de los caracteres morfológicos y demográficos (Morales, 2000). La hipótesis que deseaba probar en ese estudio era que los atributos morfológicos tenderían a diferenciarse más de sus ancestros, asimismo, observaría más diversificación en especies anuales que en especies perennes. Este tipo de análisis también puede considerarse como una estimación de la inercia filogenética. Menos cambios en el valor del atributo nos indicaría que éste no ha variado considerablemente a lo largo de su historia evolutiva. De los catorce caracteres analizados, sólo observé pocos cambios entre los valores estimados para los ancestros y los valores actuales.

Los atributos demográficos presentan un menor número de cambios, lo que implica más restricciones históricas para esos caracteres. Cuando consideré las diferencias entre especies anuales y perennes, el mayor número de cambios estaba asociado a las especies anuales. Los resultados sólo apoyan parcialmente las hipótesis propuestas.

\section{Discusión}

El Método Comparativo ha sido ampliamente utilizado en los últimos 10 años, pero también ha sido severamente criticado por su capacidad para inferir adaptaciones (Frumhoff y Reeve 1994, Leroi et al. 1994, Doughty 1996). Sin embargo, críticas sobre su validez estadística y su aplicación para entender problemas relacionados con procesos evolutivos no han surgido de una manera tan vehemente. En mi opinión, el análisis de la adaptación desde una perspectiva del método comparativo es un problema de enfoque; el método comparativo no está diseñado para entender el proceso de adaptación. Podemos inferir evolución convergente y divergente, evolución correlacionada entre pares de caracteres, estimar la inercia filogenética, pero hasta ahora sólo Hansen (1997) ha intentado formalizar un enfoque comparativo para analizar la adaptación. Un problema central en el empleo del método comparativo es que al momento no tenemos un modelo único (discutiré esto más adelante en esta sección) y no contamos con un método para el análisis multivariado de caracteres.

Como cualquier modelo que resulta novedoso, el método comparativo puede ser "víctima" de uso y abuso para tratar una amplia variedad de problemas en biología evolutiva. Considero importante subrayar que los análisis estadísticos convencionales pueden ser igualmente informativos que los análisis comparativos en función de la información genealógica disponible. Un ejemplo de lo anterior es el empleo de los análisis de varianza anidados para evaluar a qué nivel taxonómico se explica el mayor porcentaje de varianza de un atributo fenotípico en ausencia de una hipótesis filogenética. Los resultados en este caso, serían comparables al análisis de autocorrelación (p.ej. Cheverud et al. 1985) y podrían interpretarse también como la estimación de la inercia filogenética.

En la sección anterior mencioné que el análisis de contrastes independientes (Felsenstein 1985), es probablemente el enfoque comparativo más utilizado en ecología vegetal y cuyo empleo es el que más se ha discutido y comparado con los resultados obtenidos a partir de análisis de correlación convencionales sin considerar a las especies como puntos independientes. Franco y Silvertown (1996) y Ackerly y Donoghue (1998), son los únicos ejemplos de autores que han comparado los coeficentes de correlación de los análisis de contrastes y los obtenidos con los análisis de 
correlación. Franco y Silvertown (1996), reportan que la gran mayoría de las correlaciones obtenidas al comparar los atributos fenotípicos sin ninguna transformación, se mantienen al incorporar la taxonomía o la filogenia al análisis. Ackerly y Donoghue (1998), van un poco más adelante y correlacionan los coeficientes de correlación resultado con el análisis de contrastes y los obtenidos de los datos "crudos"; aunque no muestran un estadístico los valores de ambos coeficientes se observan muy similares (figura $7 \mathrm{a}, \mathrm{pp}: 785$ en Ackerly y Donoghue 1998). Posteriormente, obtienen el valor absoluto de la diferencia de ambos coeficientes como una medida de la discrepancia entre los análisis de contrastes y de correlación convencional. Los autores señalan que para atributos con más homoplasia, ambos enfoques son muy similares y concluyen recomendando que cualquier información filogenética disponible debe de ser incorporada en análisis comparativos.

La polémica sobre el uso del procedimiento de contrastes ha sido sostenida por Westoby et al (1995.) y alentada recientemente por Mazer (1998), quienes consideran que para algunos autores (principalmente Harvey et al. 1995 a y b) este enfoque es el único que se puede aplicar y pareciera que su uso se ha vuelto automático y obligatorio, además de que "obscurece" patrones evolutivos reales. Westoby et al (1995), anotan que su desacuerdo con estos autores, no es debido al análisis de correlación como tal, sino a las interpretación de los resultados. Señalan también que el análisis de contrastes es considerado como la opción correcta y que el análisis de valores fenotípicos "observados", puede conducir a correlaciones espurias debido a que las especies comparten valores similares en los atributos fenotípicos por descendencia y no adaptación; lo que genera pseudoreplicación. Concluyen indicando que no existe un procedimiento estadístico único para todas las preguntas y por otra parte, los procedimientos análiticos no deben ser estigmatizados como incorrectos sin antes analizar con detenimiento las conclusiones derivadas de ellos. Las interpretaciones son correctas o incorrectas, no los procedimientos estadísticos como tales. Con la evidencia anterior resulta claro que los análisis empleados por Westoby y colaboradores para entender procesos ecológicos y eventos de adaptación son principalmente análisis de correlación convencionales y análisis de varianza. Cabe mencionar que el grupo de investigación de Westoby ha generado información muy relevante en el área de ecología y evolución del tamaño de la semilla.

Mazer (1998), ha efectuado una crítica al análisis de contrastes en el mismo sentido que Westoby et al. (1995) al revisar la publicación de la reunión Plant life histories: ecological correlates and phylogenetic constraints. Considera que la diferencia entre los análisis de contrastes y los convencionales no es estadística, sino la forma en que los patrones pueden ser utilizados para inferir la acción de los procesos. Argumenta que con el análisis de contrastes al mostrarse evidencia de correlación evolutiva entre dos o más caracteres, se maneja el resultado como la única evidencia de un proceso evolutivo. Asimismo, menciona que la ausencia de cambio de un carácter entre especies, es necesariamente el resultado de inercia filogenética. Concluye que el análisis de contrastes se basa en sólo un enfoque de cómo los patrones pueden mostrar procesos evolutivos, lo cual no es adecuado en todas las situaciones.

Considero que este último argumento es cierto particularmente en ausencia de una filogenia, pero su propia conclusión es contradictoria al reconocer que el análisis de contrastes es sólo un enfoque particular en los estudios de biología evolutiva. La polémica puede parecer exagerada y no parece estar aportando elementos novedosos en el perfeccionamiento o refinamiento de los análisis comparativos. Estamos cerca de una discusión espuria del tipo "mi método es el mejor". Creo que mientras existan más comparaciones con diferentes enfoques podremos discutir los procesos evolutivos en ecología vegetal. Estoy convencido que el análisis de contrastes no es la panacea y como metodología puede emplearse en exceso y llevarnos a conclusiones equivocadas, sobre todo por un empleo indiscrimando y no poner atención en sus supuestos. Sin embargo, las reflexiones y las consideraciones que podemos hacer al encontrar resultados que apoyen o contradigan la teoría ecológica, son la principal virtud de estos análisis. En mi opinión el análisis de contrastes es, efectivamente, un enfoque más, pero al complementarlo y compararlo con los análisis convencionales, puede generar una discusión muy motivadora en ecología vegetal. Es claro, con base en lo anterior, que no estoy a favor de la dicotomía análisis de contraste vs análisis convencionales conforme lo plantean Westoby et al. (1995) y Mazer (1998); prefiero un enfoque complementario.

Ricklefs y Stark (1996), Price (1997) y Garland et al., 1999, también han puesto particular atención a esta polémica y aunque su trabajo se ha enfocado al estudio de vertebrados, su postura sobre el uso de correlaciones con valores fenotípicos y correlaciones con contrastes es importante para la discusión general sobre el método comparativo.

Ricklefs y Stark (1996), recopilaron los resultados de trece estudios de vertebrados, principalmente aves y mamíferos y compararon los coeficientes de correlación de los análisis de contrastes y los convencio- 
nales de regresión sin considerar la filogenia. Los coeficientes son bastante similares (no reportan ningún estadístico) o ligeramente menores para el análisis de contrastes, debido a las derivaciones empleadas en la estimación. La consistencia entre ambos coeficientes es debida a que estiman las mismas tendencias de diversificación y emplean la misma información y los resultados pueden variar sólo cuando dichos patrones de diversificación varían en la historia de los grupos. Consideran que este tipo de análisis comparativos continuarán desarrollándose y aplicándose, pero no debemos olvidar las comparaciones convencionales considerando los valores fenotípicos. Concluyen que es conveniente promover el que para realizar un análisis comparativo se elijan taxa en función de los estimados de sus relaciones filogenéticas.

El análisis de contrastes independientes ha tenido un impacto importante en biología evolutiva en general y ha permitido que reconsideremos ciertas conclusiones que se habían obtenido en investigaciones anteriores, pero también y de una manera muy importante ha confirmado las conclusiones de muchas investigaciones que no contemplaban la información filogenética en sus análisis. Creo que es importante actuar con precaución ante esta evidencia. Los análisis de contrastes necesitan por definición que incorporemos las relaciones filogenéticas de las especies a comparar y desafortunadamente no siempre podemos obtener esa información, lo cual limita el número de análisis que podemos hacer con ese procedimiento. Considero que existe la tendencia a promover el uso de la técnica de contrastes sobre los análisis convencionales, sobre todo por su rigor estadístico, sin embargo; debemos de evitar este sesgo. La información debe ser analizada con estadística convencional y con análisis comparativos. Las diferencias encontradas, de existir, deben de ser consideradas informativas y ayudarnos en la obtención del análisis correcto, área que necesita todavía numerosos modelos y sus respectivas pruebas, para poder generar "el método comparativo" que pueda ser empleado bajo cualquier evidencia de relaciones genealógicas, llámese taxonomía o filogenia.

El impacto del método comparativo en ecología vegetal ha sido importante también por el hecho del creciente interés por entender qué proporción de los atributos fenotípicos, entre taxa, está determinado por la convergencia evolutiva, y cuál es debida a tener un ancestro común. Lo anterior se refiere a lo que aquí he llamado la inercia filogenética y es también un interesante punto de discusión entre los investigadores que aplican análisis estadísticos convencionales y quienes emplean metodologías que incluyen las relaciones filogenéticas.
La estimación de la inercia filogenética con los modelos de autocorrelación hasta el momento no ha sido muy empleada en ecología vegetal, principalmente porque los análisis de varianza (anidados, jerárquicos, de covarianza) convencionales pueden proporcionarnos información muy similar. Además, para realizar un análisis de varianza, no se necesita incorporar las relaciones filogenéticas de las especies a comparar, basta con la información taxonómica de las especies. Lo anterior hace que los análisis de varianza sean más comunmente empleados porque las reconstrucciones filogenéticas aún se encuentran en una etapa de desarrollo y el número de taxa para los que tenemos esa información se podría decir que es reducido. En particular, los análisis de varianza analizan cómo se encuentra distribuida la varianza de un atributo fenotípico en diferentes niveles taxonómicos. El análisis de autocorrelación (Cheverud et al. 1985), nos permite analizar también ese componente, sin embargo va un poco más allá al determinar que no toda la variación en el carácter se encuentra incluida en la estructura de relaciones genealógicas sino que tiene un componente de varianza asociado a la evolución ulterior del carácter.

Un ejemplo de los estudios que emplean análisis de varianza son los trabajos de Mazer (1989), Herrera (1992), Lord et al (1995) y Franco y Silvertown (1996). En todos los estudios se encontró gran influencia de la estructura taxonómica en los atributos evaluados. Por ejemplo, (Mazer 1989) mostró que el género explica el $71 \%$ de la varianza total en el tamaño de la semilla de la flora de las dunas de Indiana, EUA. Herrera (1992), encontró que el género y la especie indicaban entre el $20 \%$ y el $50 \%$ de la varianza total de la longitud y el ancho de los frutos. Lord et al (1995), analizando el tamaño de la semilla en seis diferentes floras reportan que el $83 \%$ de la varianza total en el tamaño de la semilla se explicaba dentro del nivel subclase. Franco y Silvertown (1996), explican qué atributos demográficos dependientes de la edad (p.ej. edad a la madurez sexual) concentran su variación al nivel división y los caracteres dependientes del tiempo (p.ej. tasa reproductiva neta) la presentan a nivel especie. Lo anterior muestra un importante componente filogenético en atributos de los frutos, las semillas y caracteres demográficos, pero la gran pregunta que surge de estas comparaciones sigue siendo ¿cómo debemos interpretar estos patrones? Uno de los enfoques más comunes ha sido el darle una explicación adaptativa. Cuando se propuso el modelo de autocorrelación (Cheverud et al. 1985), se consideró que el análisis por sí mismo no podía proporcionarnos evidencia de adaptación; análisis posteriores de los residuales (valores 
específicos del modelo) podrían sugerir alguna evidencia de adaptación. Sin embargo, este ha sido el punto más conflictivo en los análisis del modelo de autocorrelación. Westoby et al. (1995), sostienen que el procedimiento de "corrección filogenética" considera a las explicaciones filogenéticas y ecológicas como mutuamente excluyentes y da prioridad al componente filogenético, ubicando a la ecología como una última opción que es tomada en cuenta sólo cuando la hipótesis filogenética no se fundamenta. Concluyen que emplear un enfoque estadístico convencional permite formular las preguntas e hipótesis en términos de patrones actuales más que en términos de los origenes históricos.

Considero que las propuestas del grupo de Westoby, en términos de qué enfoque analítico emplear para realizar comparaciones, en cierto modo están sesgadas y no totalmente fundamentadas. Reconozco que el uso del método comparativo se ha difundido de una manera importante y para algunos autores su empleo es prioritario y en ocasiones el único análisis posible. Sin embargo, el análisis de autocorrelación no ha sido derivado para ser la única alternativa para analizar la información. De hecho, es un análisis que nos permite ir un poco más allá que los análisis de varianza, porque éstos descomponen el 100\% de la variación de los atributos fenotípicos a través de los niveles taxonómicos empleados. El análisis de autocorrelación (Cheverud et al. 1985) nos indica qué porcentaje de la varianza no está determinado por la estructura genealógica y con las modificaciones de Gittleman y Kot (1990), podemos determinar a qué nivel de la filogenia se encuentra explicada la mayor fracción de la varianza de los atributos analizados. Sin embargo no considero que ambos tipos de análisis sean opciones alternativas, son más bien complementarias y además es conveniente insistir que el análisis de autocorrelación no fue generado para eliminar los análisis convencionales, sino para realizar análisis comparativos cuando tenemos información más detallada de las relaciones filogenéticas. Precisamente no creo que la mejor opción sea incluir las relaciones filogenéticas para cualquier tipo de análisis, puesto que existe una gran cantidad de preguntas que necesitamos responder en ecología y muchas de ellas no necesariamente requieren la inclusión de éstas. Pero estoy totalmente seguro de que incluir las relaciones filogenéticas en nuestros análisis comparativos, nos permite tener un espectro de preguntas igual o bastante más amplio. No creo que debamos alentar esta polémica, pues es muy claro que ambos métodos están intentando responder preguntas similares con diferentes enfoques. Lo que considero que debemos hacer es analizar la información con diferentes métodos y buscar coincidencias y resaltar diferencias, para poder obtener una metodología unificadora en biología evolutiva.

El aspecto que más se discute en torno a la aplicación del método comparativo es su capacidad para determinar el valor adaptativo de los atributos fenotípicos comparados. Una de las principales críticas al uso del método comparativo es que el empleo de caracteres y ambientes con las distribuciones que presentan actualmente no pueden ser empleadas para determinar los mecanismos de cambio evolutivo y más aún, que los patrones filogenéticos pueden sugerir que un carácter pueda ser una adaptación cuando no lo es y viceversa (Leroi et al. 1994). También sugieren que emplear estos métodos de una manera muy aislada sólo puede proporcionar evidencias muy limitadas de los mecanismos y procesos evolutivos. Considero que estos argumentos tienen un importante sesgo porque como ya he mencionado, los análisis comparativos no han sido formulados para el estudio de la adaptación, pero sí pueden ser útiles para hacer inferencias sobre el valor adaptativo de los caracteres. En general, cuando empleamos el método comparativo lo hacemos en combinación con otras técnicas que nos permitan evaluar hipótesis evolutivas y entender el significado funcional de los caracteres como los observamos actualmente y los procesos históricos bajo los que han evolucionado.

Los análisis comparativos se basan en hipótesis filogenéticas, que son sólo eso y con esa cautela deben tratarse. El método comparativo emplea filogenias que los investigadores suponen que no presentan "errores" y las imprecisiones en éstas, de existir, tienen efecto la credibilidad de los resultados obtenidos. Afortunadamente los métodos de reconstrucción filogenética han sido criticados y revisados por los taxónomos durante los últimos años, lo cual redundará en filogenias más precisas que serán empleadas en análisis comparativos. Es importante también tener un amplio conocimiento de la biología de las especies que queremos comparar, lo cual hace necesario que colaboremos de manera muy cercana con los investigadores en sistemática.

Los métodos comparativos son una herramienta excelente para estudiar la evolución de caracteres, sobre todo porque muchas explicaciones evolutivas no pueden ser sujeto de experimentación o manipulación. Sin embargo, debido a que se trata de herramientas estadísticas, debemos ser muy cuidadosos para "cumplir" con todos los supuestos del modelo antes de postular cualquier conclusión. El método comparativo sigue desarrollándose (Pagel 1997 y Garland et al., 2000) y puede aplicarse para inferir los procesos históricos del pasado, dados los patrones de diversi- 
dad de los caracteres en el presente. Un aspecto que todavía es limitante en el empleo del método comparativo es la falta de modelos para realizar análisis multivariados.

Para finalizar esta sección, quisiera añadir que el método comparativo es de gran utilidad en el estudio de evolución de caracteres, sin embargo, todavía es necesario mucho trabajo en esta área, en términos de desarrollar modelos y probar los que ya existen con datos no obtenidos de simulaciones, sino con datos de la variación que existe en la naturaleza. Considero que es muy importante promover el empleo de esta metodología en biología evolutiva y en otras áreas de la biología.

\section{Conclusiones}

Las comparaciones intraespecíficas siempre han sido y seguirán siendo una herramienta esencial en los estudios de ecología comparativa y evolutiva. El método comparativo ha permitido un mayor rigor en las explicaciones evolutivas realizadas a partir de información ecológica y/o morfológica. Existen ejemplos en los que las conclusiones obtenidas pueden modificarse al usar un análisis comparativo (p.ej. Kelly y Purvis 1993), pero también existen ejemplos en lo que esto no sucede (ver Silvertown y Dodd 1996). Lo anterior hace necesario que continuemos realizando comparaciones para determinar el impacto de este tipo de análisis. Los análisis que incluyen información filogenética continuarán desarrollándose y aplicándose, pero también es necesario considerar que los análisis convencionales no deben excluirse y de ser posible combinarse con los análisis comparativos para evaluar hipótesis evolutivas.

Es claro que el método comparativo no es el único modelo para aceptar o rechazar hipótesis en biología evolutiva, sin embargo, tiene un potencial enorme para responder diferentes preguntas.

\section{Agradecimientos}

Quiero agradecer a Erika Aguirre la revisión crítica del presente trabajo en todas sus fases y sus sugerencias que mejoraron sustancialmente el presente trabajo. A dos revisores anónimos por sus comentarios. a Rafael Lira, editor del Boletín por su paciencia y al CONACyT por financiar el presente trabajo.

\section{Literatura Citada}

Abrahamson W. G. 1979. Patterns of resource allocation in wildflower populations of fields and woods. American Journal of Botany 66:71-79.

Ackerly D. D. y Donoghue M. J. 1998. Leaf size, sapling allometry, and Corner's rules: phylogeny and correlated evolution in maples (Acer). American Naturalist 152:767-791.

Armstrong D. P. y Westoby M. 1993. Seedlings from large seeds tolerate defoliation better: a test using phylogenetically independent contrasts. Ecology 74:1092-1100.

Beerling D. J. y Kelly C. K. 1996. Evolutionary comparative analyses of relationship between leaf structure and fuction. The New Phytologist 134: 35-51.

Brooks D. R. y McLennan D. 1991. Phylogeny, ecology and behavior: a research program in comparative biology. University of Chicago Press. Chicago. 434 p.

Coddington J. A. 1998. Cladistics tests of adaptational hypotheses. Cladistics 4:3-22.

Crawley M. J., Harvey P. H. y Purvis A. 1996. Comparative ecology of the native and alien floras of the British Isles. Philosophical Transactions of the Royal Society of London Series B 351:1251-1260.

Cunningham C. W., Omland K. E. y Oakley T. H. 1998. Reconstructing ancestral character states: a critical reappraisal. Trends in ecology and evolution 13:361-366.

Cheverud J. M., Dow M. M. y Leutenegger W. 1985. The quantitative assesment of phylogenetic constraints in comparative analyses: sexual dimorphism in body weight among primates. Evolution 39:1335-1351.

Diniz-Filho J. A. F., Ramos de Sant'Ana C. E. y Bini, L. M. 1998. An An eigenvector method for estimating phylogenetic inertia. Evolution 52: 1247-1262.

Doughty P. 1996. Statistical analysis of natural experiments in evolutionary biology: Comments on recent criticisms of the use of comparative methods to study adaptation. American Naturalist 148:943-956.

Ezcurra C., Ruggiero, A. y Crisci, J. V. 1997. Phylogeny of Chuquiraga sect. Acanthophyllae (Asteraceae-Barnadesioideae), and the evolution of its leaf morphology in relation to climate. American Journal of Botany 22:151-163.

Feeny P. P. 1976. Plant apparency and chemical defense. Recent Advances in Phytochemistry 10:1-40.

Felsenstein J. 1985. Phylogenies and the comparative method. American Naturalist 125:1-15.

Foster S. A. y Janson C. H. 1985. The relationship between seed size and establishment conditions in tropical woody plants. Ecology 66:773-780.

Franco M. y Silvertwon J. 1996. Life-history variation in plants: an exploration of the fast-slow continum hypothesis. Philosophical Transactions of the Royal Society of London Series B 351:1341-1348.

Frumhoff P. C. y Reeve H. K. 1994. Using phylogenies to test hypothesis of adaptation: a critique of some current proposals. Evolution 48:172-180.

Garland T. Jr., Midford P. E. e Ives A. R. 1999. An Introduction to phylogenetically based statistical methods, with a new method for confidence intervals on ancestral values. American Zoologist 39:374-388.

Gittleman J. L. y Kot M. 1990. Adaptation: statistics and a 
null model for estimating phylogenetic effects. Systematic Zoologist 39: 227-241.

Givnish T. J. 1987. Comparative studies of leaf form: assessing the relative roles of selective pressures and phylogenetic constraints. New Phytologist 106:S131-S160.

Hansen T. F. 1997. Stabilizing selection and the comparative analysis of adaptation. Evolution 51:1341-1351.

Harvey P.H. y Pagel M. D. 1991. The comparative method in evolutionary biology. Oxford University Press. Oxford. 239 p.

Harvey P. H., Read A. F. y Nee S. 1995 a. Why ecologists need to be phylogenetically challenged. Journal of Ecology 83:535-536.

Harvey P. H., Read A. F. y Nee S. 1995 b. Further remarks on the role of phylogeny in comparative ecology. Journal of Ecology 83:733-734.

Herrera C. M. 1992 Interspecific variation in fruit shape: allometry, phylogeny, and adaptation to dispersal agents. Ecology 73:1832-1841.

Jordano P. 1995. Angiosperm fleshy fruits and seed dispersers: a comparative analysis of adaptation and constraint in plant-animal interactions. American Naturalist 145:163191.

Kelly C. K. 1995. Seed size in tropical trees: a comparative study of factors affecting seed size in Peruvian angiosperms. Oecologia 102:377-388.

Kelly C. K. y Purvis A. 1993. Seed size and establishment conditions in tropical trees: on the use of taxonomical relatedness in determining ecological patterns. Oecologia 94:356-360.

Kelly C. K. y Beerling D. J. 1995. Plant life form, stomatal density, and taxonomic relatedness: a reanalysis of Salisbury (1927). Functional Ecology 9:422-431.

Kelly C. K. y Woodward F. I. 1995. Ecological correlates of carbon isotope composition: a comparative analysis testing for the effects of temperature, $\mathrm{CO}_{2}$ y $\mathrm{O}_{2}$ partial pressure and taxonomic relatedness on $\delta^{13} \mathrm{C}$. Journal of Ecology 83:509-515.

Kelly C. K. y Woodward F. I. 1996. Ecological correlates of plant range size: taxonomic and phylogenies in the study of plant commonness and rarity in Great Britain. Philosophical Transactions of the Royal Society of London Series B 351:1261-1269.

Koshi J. M. y Goldstein R. A. 1996. Probabilistic reconstruction of ancestral protein sequences. Journal of Molecular Evolution 42:313-320.

Leroi A. M., Rose M. R. y Lauder G. V. 1994. What does the comparative method reveal about adaptation? American Naturalist 143:381-402.

Lord J., Westoby, M. y Leishman M. (1995). Seed size and phylogeny in six temperate floras: constraints, niche conservatism, and adaptation: American Naturalist 146:349364.

Maddison W.P. (1995). Calculating the probability distributions of ancestral states reconstructed by parsimony on phylogenetic trees. Systematic Biology 44:474-481.

Martins E. P. (1994). Estimating the rate of phenotypic evolution from comparative data. American Naturalist 144:193-209.

Martins E. P. y Hansen T. (1996). The statistical analysis of interespecific data: a review and evaluation of phylogenetic comparative methods. En: E. Martins Ed. Phylogenies and the comparative method in animal behavior. Oxford Univesity Press, Oxford, 22-75.

Martins E. P. y Hansen T. (1997). Phylogenies and the comparative method: a general approach to incorporating phylogenetic information into the analysis of interspecific data. American Naturalist 149:646-667.

Mazer S. J. 1989. Ecological, taxonomic and life history correlates of seed mass among Indiana dune angiosperms. Ecological Monographs 59:153-175.

Mazer S. J. 1998. Alternative approaches to the analysis of comparative data: compare and contrast. American Journal of Botany 85: 1194-1199.

Miles D.B. y Dunham A.E. 1993. Historical perspectives in ecology and evolutionary biology: the use of phylogenetic comparative analyses. Annual Review of Ecology and Systematics 24:587-619.

Morales E. 1996. El Método comparativo en los estudios de evolución de historias de vida: un ejemplo con el género Tithonia (Asteraceae). Tesis doctoral, UNAM, México, $101 \mathrm{pp}$.

Morales, E. 2000. Estimating phylogenetic inertia in the genus Tithonia (Asteraceae): A comparative approach. Evolution 54:

Pagel M. D.1997. Inferring evolutionary processes from phylogenies. Zoologica Scripta 26:331-348.

Pagel M. D. y Harvey P.H. 1988. Recent developments in the analysis of comparative data. The Quarterly Review of Biology. 63:413-440.

Price T. 1997. Correlated evolution and independent contrasts. Philosophical Transactions of the Royal Society of London Series B 352:519-529.

Ress M. 1996. Evolutionary ecology of seed dormancy and seed size. Philosophical Transactions of the Royal Society of London Series B 351:1299-1308.

Ricklefs R. E. y J. M. Starck 1996. Applications of phylogenetic independent contrasts: a mixed progress report. Oikos 77:167-172.

Rose M.R. y Lauder G. V. 1996. Adaptation. Academic Press. San Diego. 511 p.

Ridley M. y Grafen A. 1996. How to study discrete comparative methods. En: E. Martins Ed. Phylogenies and the comparative method in animal behavior. Oxford Univesity Press, Oxford. 76-103.

Salisbury E. J. 1927. On the causes and ecological significance of stomatal frecuency, with special reference to the woodland flora. Philosophical Transactions of the Royal Society of London Series B 216:1-65. 
Schluter D. 1995. Uncertainty in ancient phylogenies. $\mathrm{Na}$ ture 377:108-109.

Schluter D., Price T., Mooers A. O. y Ludwig D. 1997. Likelihood of ancestor states in adaptive radiation. Evolution 51:1699-1711.

Silvertown J y Dodd M. 1996. Comparing plants and connecting traits. Philosophical Transactions of the Royal Society of London Series B 351:1233-1239.

Wanntorp H-E. 1983. Historical constraints in adaptation theory: traits and no traits. Oikos 41:157-160.

Westneat M.W. 1995. Feeding, function, and phylogeny: analysis of historical biomechanics in labrid fishes using comparative methods. Systematic Biology 44:361-383. Westoby M., Leishman M. R. y Lord J. M. 1995. On missinterpreting the 'phylogenetic correction'. Journal of Ecology 83:531-534.

Yang Z., Kumar S y Nei M. 1995. A new method of inference of ancestral nucleotide and amino acid sequences. Genetics 141:1641-1650.

Zhang J., y Nei M. 1997. Accuracies of ancestral amino acid sequences inferred by the parsimony, likelihood, and distance methods. Journal of Molecular Evolution S139-S146. 